\title{
Penile Melanotic Macules: A Case Report
}

\author{
Rosa Gimenez-Garcia*, Sergio Carrasco Molina \\ Department of Dermatology, Hospital Rio Hortega, Valladolid, Spain
}

*Corresponding Author: Rosa Gimenez-Garcia, Department of Dermatology, Hospital Rio Hortega, Valladolid, Spain, Email: rosagim@hotmail.com

\begin{abstract}
Penile melanoticmacules, a benign acquired melanosis generally observed in adulthood, may be present on the shaft of the penis or on the glans penis and can mimic mucosal melanoma. Different dermoscopic patterns has been identified in cases of mucosal melanosis but there are few reported cases about findings in male melanosis

We report a case of penile melanosis on the fore skin of the penis in a 41-year-old male with personal history of lichen sclerosus on the glans. Dermoscopic examination revealed parallel pattern.

Melanotic macules are observed on the mouth, genitalia and the nails. Penile melanosis present as multifocalor variegated pigmented macular lesions are often misdiagnosed as melanoma. Histologically no melanocytic proliferation nor melanocytic atypia are shown. Melanotic macules may occur as a part of sindromes with multisystem anomalies as Peutz-Jeghers syndrome, Carney complex, LEOPARD syndrome, LAMB syndrome; NAME sindrome or Ruvalcaba-Myhre syndrome. Dermoscopy it is a noninvasive and useful diagnostic tool for study of genital melanosis. The most frequent pattern are the parallel pattern and ring- like pattern followed by globular, reticular or mixed patterns.
\end{abstract}

Keywords: melanosis penis; penile macules.

\section{INTRODUCTION}

Genital melanosis are uncommon. [1] Penile melanotic macules, a benign acquired melanosis generally observed in adulthood, may be present on the shaft of the penis or on the glans penis and can mimic mucosal melanoma.[2-5] Different dermoscopic patterns has been identified in cases of mucosal melanosis but there are few reported cases about findings in male melanosis

\section{CASE Report}

A 41-year-old male with personal history of lichen sclerosus on the glans presented to us with hyperpigmentation on the foreskin of the penis. (Fig1) On dermoscopic examination revealed parallel pattern. (Fig2) Patient was circumcised. Histo pathological study demonstrated hyper pigmentation of basal keratinocytes with neither hyperplasia nor atypical melanocytes consistent with diagnosis of melanotic macules of the penis.

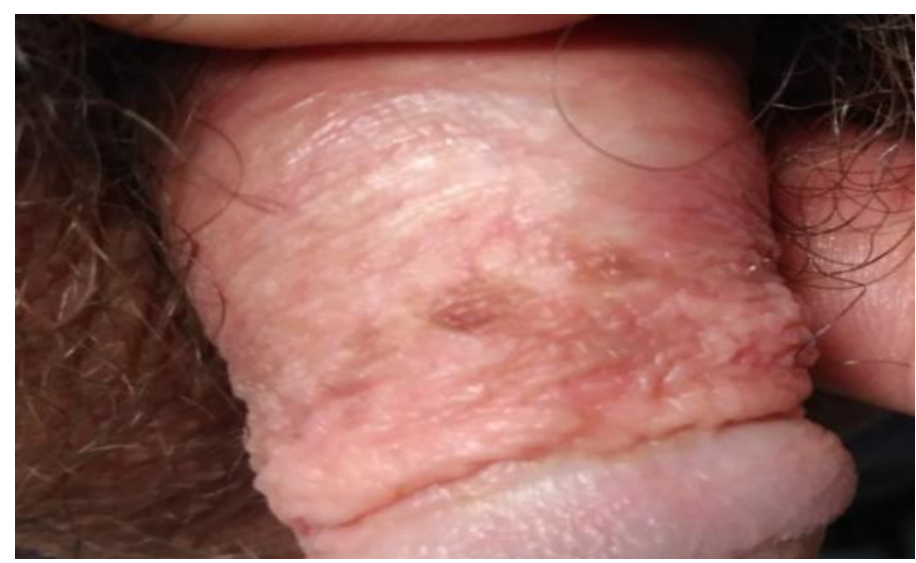

Fig1.Multifocal irregular macules with variegated pigmentation 

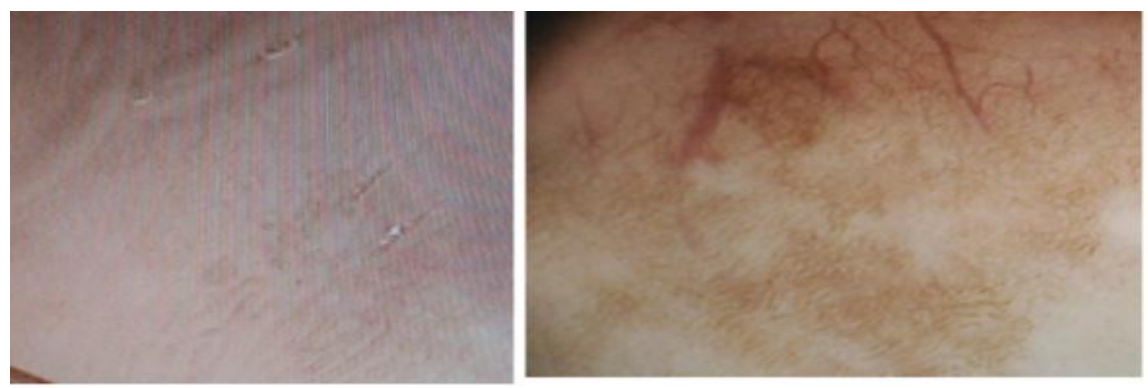

Fig2. Dermoscopic examination showing parallel pattern (uniform linear and curved structures)

\section{DISCUSSION}

Melanotic macules are observed on the mouth, genitalia and the nails. Penile melanosis is a condition clinically characterized by multifocal or variegated pigmented macular lesions of irregular colour are often misdiagnosed as melanoma. Because pigmented lesions on mucous membranes and anogenital skin are atypical morphologically, they should be biopsied. Melanotic macules may occur as a part of syndromes with multisystem anomalies as Peutz-Jeghers syndrome, Carney complex, LEOPARD syndrome, LAMB syndrome; NAME syndrome or Ruvalcaba-Myhresyndrome. The diagnosis must be confirmed by histophato logical study. Histopathology of penile melanosis or lentiginosis is characterized by an increase in melanin in the basal layer, a discrete melanocytic hyperplasia without nest formation, as well as an epithelial hyperplasia and melanophages in the dermis. [1-5]

There are only a few case reports about genital melanosis in literature and little is described about dermoscopic features of genital melanosis. Dermoscopy it is a noninvasive anduseful diagnostic tool for study of genital melanosis. The most frequent pattern are the parallel pattern and ring- like pattern followed by globular, reticular or mixed patterns. [6-9]

We have found only a previously reported case of penile melanosis associated with lichen sclerosus. [10]

\section{CONCLUSION}

Penile melanosis is quite common and may mimic malignant melanoma. Dermoscopic analysis can improve accuracy in diagnosing pigmented penile skin lesions. Genital melanosis demonstrate rign like, parallel, structurless or reticular-like dermoscopic patterns differing from malignant melanoma.

\section{REFERENCES}

[1] Lenane P, Keane CO, Connell BO, Loughlin SO, Powell FC. Genital melanotic macules: clinical, histologic, immunohistochemical, and ultrastructural features. J Am Acad Dermatol. 2000 Apr; 42(4):640-4.

[2] Jairath V, Jindal N, Sehrawat M, Jain VK, Kaur S, Priyadarshini MM. Benign penile melanosis: A linear variant. Indian J Dermatol Venereol Leprol. 2015 Nov-Dec; 81(6):655.

[3] Cervigón I, Palomo A, Torres LM. Melanotic macules of the penis. Actas Dermosifiliogr. 2007 Dec;98(10):722-3

[4] Laguna C, Pitarch G, Roche E, Fortea JM. A typical pigmented penile macules. Actas Dermosifiliogr. 2006 Sep; 97(7):470-2.

[5] Giménez GR, Olmosa A, Sánchez S, Sanz C. Melanosis del pene: a propósito de dos casos. Med CutanIberLat Am 2003; 31 (6)

[6] Mannone F, De Giorgi V, Cattaneo A, Massi D, De Magnis A, Carli P. Dermoscopic features of mucosal melanosis. Dermatol Surg. 2004; 30: 1118-1123.

[7] Cengiz FP, Emiroglu N, Wellenhof RH. Dermoscopic and clinical features of pigmented skin lesions of the genital area. An Bras Dermatol. 2015 Mar-Apr;90(2):178-83

[8] Ferrari A, Buccini P, Covello R, De Simone P, Silipo V, Mariani G, Eibenschutz L, Mariani L, Catricalà C. The ring like pattern in vulvar melanosis: a new dermoscopic clue for diagnosis. Arch Dermatol. 2008;144:1030-4

[9] Ronger-Savle S, Julien V, Duru G, Raudrant D, Dalle S, Thomas L. Features of pigmented vulval lesions on dermoscopy. Br J of Dermatol. 2010; 164: 54-61.

[10] Tchernev G, Chokoeva AA, Mangarov H. Penile Melanosis Associated with Lichen Sclerosus et Atrophicus: First Description in the Medical Literature. Open Access Maced J Med Sci. 2017 Aug 10;5(5):692-693

Citation: Rosa Gimenez-Garcia, Sergio Carrasco Molina, Penile Melanotic Macules: A Case Report. ARC Journal of Dermatology. 2018; 3(3):1-2. doi:dx.doi.org/10.20431/2456-0022.0303001.

Copyright: () 2018 Authors. This is an open-access article distributed under the terms of the Creative Commons Attribution License, which permits unrestricted use, distribution, and reproduction in any medium, provided the original author and source are credited. 